\title{
L'INTERFÉRENCE CHEZ LES ÉTUDIANTS EN PHILOLOGIE ITALIENNE EN TANT QUE DÉFI POUR L’ENSEIGNANT
}

\begin{abstract}
Karczewska Małgorzata, L'interférence chez les étudiants en philologie italienne en tant que défi pour l'enseignant [Linguistic interference occurring in students of Italian philology as a challenge for a lecturer], Studia Romanica Posnaniensia, Adam Mickiewicz University Press, Poznań, vol. XXXVIII/2 : 2011, pp. 35-46. ISBN 978-83-232-2335-1. ISSN 0137-2475. DOI 10.2478/v10123-011-0012-z.
\end{abstract}

Polish students of Italian philology in Poznań study two Romance languages: Italian and French, which on one hand facilitates the process of learning, as many rules of grammar and words are similar in both languages. On the other hand, though, the closeness of two linguistic systems is the source of the interference phenomenon even if none of the languages is a mother tongue. As students of philology are expected to speak perfectly the foreign language studied, all mistakes need to be eliminated. In the present paper we concentrate on mistakes made in Italian due to the interference of French. After a short theoretical introduction on interference we give some examples of errors to illustrate the phenomenon and to show the areas in which errors are particularly numerous. In the third part of the paper we suggest some solutions which are based on observations and conclusions resulting from the professional experience as a lecturer in Italian.

\section{INTRODUCTION AU PROBLÈME DE L'INTERFÉRENCE}

Tous ceux qui apprennent plus d'une langue étrangère et tous les enseignants de langues connaissent bien le phénomène de l'interférence linguistique. D'une part, il est plus facile de maîtriser une langue si elle appartient à la même famille que la langue déjà maîtrisée car cette préconnaissance facilite d'une manière décisive la compréhension et la mémorisation de divers éléments de langue, mais d'autre part on est, dans cette situation, plus enclin à commettre des erreurs interférentielles.

L'interférence est définie différemment par des linguistes différents puisque ce phénomène est tellement compliqué et hétérogène. La définition la plus restreinte décrit l'interférence comme l'influence négative de la langue maternelle sur la langue étrangère pendant que la définition la plus large la définit en tant qu'influence d'une langue sur une autre sans préciser ni la valeur du phénomène, ni le caractère des langues y inclues. Certains linguistes traitent comme interférence même le fait d'éviter une structure difficile dans la langue que l'on apprend. Non seulement les définitions du phénomène, mais aussi ses classements sont nombreux. On identifie l'interférence à divers niveaux de la langue en énumérant l'interférence phonique, grammaticale et lexicale qui se di- 
visent ensuite en sous-catégories (Weinreich, 1953). On peut aussi distinguer plusieurs sous-systèmes de la langue où l'interférence peut apparaître : on a l'interférence dans le sous-système intonatif, rythmique, accentuel, phonétique, morphologique, syntaxique et lexical. Une autre approche différencie enfin l'interférence sur le plan d'expression, le plan sémantique et le plan pragmalinguistique (Szulc, 1994).

Un autre classement est celui en interférence interlinguistique (qui agit au sein de la même langue) et en interférence extralinguistique (qui agit entre deux langues diverses). On distingue ensuite l'interférence positive, celle qui facilite l'apprentissage, et l'interférence négative qui le rend plus difficile. Ce qui unit la majorité des études sur l'interférence, c'est le fait que l'on examine la relation entre la langue maternelle et la langue étrangère. Par contre, dans l'étude présente, nous nous concentrons sur l'interférence entre deux langues étrangères, plus précisément entre les deux langues apprises par les étudiants en philologie italienne : la langue italienne et la langue française. Si d'une part, pour l'enseignant qui travaille avec de tels étudiants il est parfois plus facile d'introduire les questions grammaticales ou le vocabulaire en se référant aux similitudes entre les deux langues, on observe, d'autre part, chez les étudiants une grande quantité d'erreurs, surtout lexicales mais aussi grammaticales. La présente étude vise à proposer des solutions pour réduire leur nombre dans le processus de l'apprentissage et de l'enseignement. Pour ce faire, il nous est nécessaire de toute façon de connaître auparavant les mécanismes qui régissent le phénomène pour pouvoir y remédier.

La première règle de l'interférence est qu'elle agit de la structure forte, c'est-à-dire celle bien ou auparavant assimilée, vers la structure faible : nouvelle ou mal assimilée. Il faut remarquer que la force et la faiblesse concernent une structure particulière et pas nécessairement une langue entière : dans un cas c'est une structure italienne qui influence sur une structure française et dans un autre, la direction de l'influence peut être inversée.

Une autre règle, qui est très importante dans la présente étude, prétend que le nombre d'erreurs augmente quand les deux langues sont proches, semblables et diminue quand les langues sont distantes (Komorowska, 1980). Cette théorie n'est pas partagée par tous les linguistes, mais dans le cas étudié de l'italien et du français, elle est confirmée car c'est la proximité entre les deux langues qui constitue la cause principale de nombreuses erreurs. Il faut néanmoins expliquer que ni dans cette théorie, ni dans la présente étude on ne s'occupe du fait que la même proximité facilite tellement l'apprentissage : on se concentre seulement sur les erreurs et les difficultés.

L'approche psychologique explique le phénomène de l'interférence avec la généralisation de la stimulation et de la réaction. Car, en nous basant sur l'expérience de l'enseignant d'italien nous sommes plus encline à expliquer un grand nombre d'erreurs entre l'italien et le français pour deux raisons très simples :

- les étudiants sont souvent convaincus que, dans l'autre langue, l'équivalent exact du mot ou de la structure qu'ils connaissent de la première langue doit 
exister, donc il suffit d'adapter les éléments de la première langue acquise aux règles de l'autre langue pour obtenir la structure correcte,

- parfois les étudiants sont conscients des lacunes de grammaire ou lexicales qu'ils possèdent mais dans des situations d'urgence ils essayent de créer des structures sans vérifier si elles existent vraiment.

Il ne faut pas oublier que la présente étude concerne les étudiants en philologie, particulièrement doués pour les langues, qui connaissent ou, au moins, sentent intuitivement les règles de grammaire ou de formation des mots. Il est nécessaire d'ajouter que l'interférence chez les étudiants en philologie italienne était particulièrement visible à l'époque où, pour être admis à l'université, il fallait bien connaître au moins une langue romane (l'italien ou le français) qui ensuite devenait la source de l'interférence. Aujourd'hui, quand les étudiants commencent souvent à apprendre les deux langues romanes dès le début en même temps, l'impact du français sur l'italien n'est pas tellement fort. Quand même, nous observons toujours ce phénomène qui n'est pas si imprévisible que l'on pourrait penser : après quelques années d'expérience didactique nous pouvons distinguer certains domaines dans lesquels les erreurs interférentielles sont très probables. D'habitude, malgré la créativité infinie des étudiants, nous pouvons prévoir la possibilité d'erreur en comparant les deux systèmes linguistiques pour distinguer les éléments qui sont semblables mais pas identiques. En conséquence, une analyse comparée nous permet de découvrir les sources potentielles d'erreurs. Bien sûr, même si les éléments dans les deux langues sont complètement différents, l'étudiant peut essayer de créer une structure en se basant sur celle qu'il connait, mais en général c'est la similitude qui incite plus aux erreurs. Dans la présente étude, nous nous concentrons sur les erreurs dans la langue italienne commises sous l'influence de la langue française.

\section{EXEMPLE D'ERREURS COMMISES À DIVERS NIVEAUX DE LA LANGUE}

A ce stade, nous voudrions passer à la comparaison entre certaines structures des deux langues et aux exemples d'erreurs interférentielles.

\subsection{QUESTION À NIVEAU PHONÉTIQUE}

En ce qui concerne le niveau phonétique, les erreurs interférentielles n'y existent presque pas car toutes les deux langues sont étrangères pour les étudiants en question, donc nous n'observons pas cette influence caractéristique de la langue maternelle sur la prononciation dans la langue étrangère. Quand même, nous observons des erreurs où l'élément phonétique est présent. La plus grave et la plus fréquente est la prononciation du mot italien qui [kwi] (ici) à la française [ki]. Cette erreur n'est pas seulement de nature phonétique mais aussi sémantique comme beaucoup d'étudiants 
identifient le mot italien avec le sens français. En plus, la prononciation identique [ki] du vrai équivalent du qui français, le pronom italien chi, provoque encore le problème d'orthographe en italien :

\begin{tabular}{|c|c|}
\hline Mot français & Équivalent italien \\
\hline$q u i[\mathrm{ki}]$ & chi $[\mathrm{ki}]$ \\
\hline ici & $q u i[$ qui] \\
\hline
\end{tabular}

Il y a plusieurs personnes du niveau avancé du français et du niveau débutant ou intermédiaire de l'italien qui ne peuvent pas croire que la prononciation [ki] puisse correspondre à une telle orthographe en italien. L'exemple cité se trouve parmi les erreurs les plus fréquentes et les plus difficiles à éliminer. Nous reviendrons à la question de la fausse équivalence dans le paragraphe suivant.

\subsection{CATÉGORIES D’ERREURS COMMISES PAR LES ÉTUDIANTS}

En général, les erreurs des étudiants en philologie italienne causées par l'influence du français sur l'italien se divisent en six catégories :

1) l'emploi des mots français au lieu de leur équivalent italien,

2) le recours aux règles grammaticales françaises,

3) l'attribution du genre erroné aux noms italiens d'après le modèle français,

4) les fautes d'orthographe,

5) la création des mots inexistants en italien d'après le modèle français,

6) les erreurs liées à l'existence des faux amis.

Dans chaque catégorie nous avons montré le mécanisme de l'interférence sur quelques exemples l'illustrant.

2.2.1. Le problème de l'emploi des mots français dans l'énoncé italien est bien illustré par l'emploi des conjonctions mais, ou, et au lieu de leurs équivalents italiens $m a, o, e$. Pour comprendre ce phénomène, qui est pourtant très fréquent, il faut se rendre compte combien de fois l'apprenant a prononcé ces mots pendant le processus d'apprentissage et ainsi on comprendra comment ceux-ci ils sont enracinés et automatisés.

2.2.2. Le recours aux règles grammaticales françaises constitue le groupe d'erreurs le plus nombreux et varié. Nous pouvons distinguer ici d'ultérieures sous-catégories d'erreurs qui concernent différents champs de la grammaire :

a. L'emploi des articles : en général, leur emploi en français est plus ordoné et ne laisse presque aucun espace au choix alors que l'emploi des articles en italien est beaucoup plus complexe, ce qui provoque les erreurs même sans influence de la langue 
française. A titre d'exemple, nous pouvons citer l'emploi des articles partitifs : en français on dit: Je bois du café, on ne dit pas : Je bois le café alors qu'en italien on dit: Bevo il caffè ou Bevo un caffè et aussi Bevo caffè. Dans la négation en français on dit: Je ne bois pas de café pendant qu'en italien on dit : Non bevo il caffè ou Non bevo caffè sans aucun article, ce qui est impossible en français. Cette confusion rend l'étudiant pas trop sûr et peut enfin causer des erreurs également dans l'usage de la langue française. Pour conclure la question des articles, il faut ajouter qu'en italien, contrairement au français et aux autres langues, le possessif est précédé de l'article, d'habitude de l'article défini : la mia casa pour ma maison. L'erreur concerne le fait que les étudiants trouvent l'idée de précéder le possessif par l'article un peu bizarre et ils ne le font pas. Dans ce cas, ce n'est pas seulement l'influence de la langue française, mais aussi de l'anglais et d'autres langues dans lesquelles le possessif n'est pas accompagné de l'article.

À coté des difficultés dans l'emploi des articles, nous analysons l'emploi de la préposition de avec certaines expressions (p.ex. celles qui expriment la quantité) : en français on dit J'ai beaucoup d'amis alors qu'en italien on n'utilise pas di: Ho molti amici et Je n'ai pas assez d'argent contre Non ho abbastanza soldi. De la même manière, il n'y a pas $d i$ dans les expressions italiennes du type : è + adjectif + infinitif contrairement au français où l'on en a : Il est difficile de parler bien une langue étrangère contre $\grave{E}$ difficile parlare bene una lingua straniera. Il n'est pas difficile d'imaginer le nombre d'erreurs commises par les étudiants qui souvent sentent la nécessité d'ajouter $d i$ dans ces expressions en suivant le modèle français.

b. L'emploi des pronoms relatifs : en français, dans les propositions relatives, qui introduit le sujet et que introduit l'objet pendant qu'en italien chi est utilisé seulement pour remplacer le sujet de la phrase entière et pas pour l'accompagner (en français dans ce type de phrase on utilise les pronoms démonstratifs comme celui). Dans la plupart des cas, on emploie en italien le pronom che qui sert à introduire soit le sujet, soit l'objet :

\begin{tabular}{|l|l|}
\hline \multicolumn{1}{|c|}{ Phrase française } & \multicolumn{1}{c|}{ Phrase italienne } \\
\hline $\begin{array}{l}\text { C'est un homme qui parle trois langues } \\
\text { étrangères. }\end{array}$ & $\grave{E}$ un uomo che parla tre lingue straniere. \\
\hline C'est la voiture que j'ai achetée hier. & È la macchina che ho comprato ieri. \\
\hline Celui qui travaille, gagne. & Chi lavora, guadagna. \\
\hline
\end{tabular}

Dans ce cas, comme nous l'avons déjà signalé à propos de qui, nous observons aussi des erreurs d'orthographe ou au moins une certaine hésitation dans ce domaine.

c. L'accord du verbe et du participe passé avec le pronom ne et avec les constructions impersonnelles en italien, ce qui n'existe pas en français: Le birre, ne ho bevute cinque contre Les bières, $j$ 'en ai bu cinq et In Polonia si leggono molte riviste contre 
En Pologne, on lit beaucoup de magazines. Cette différence entre les deux langues et l'effort lié à l'application de l'accord peuvent inciter les étudiants à ne pas faire cet accord.

d. Le système des temps grammaticaux : en italien il n'y a pas d'équivalent du futur proche et du passé récent. Pour cette raison, on traite souvent la forme italienne de l'obligation comme la forme du futur qu'elle n'est pas : la phrase Il progetto va finito signifie qu'il faut finir le projet et pas qu'il sera fini bientôt. De la même façon, on peut créer des structures, inexistantes en français, du passif avec le verbe venir sur le modèle italien comme : *Le dîner vient préparé par maman inspiré par la phrase italienne La cena viene preparata dalla mamma qui peut provoquer elle-même encore plus de confusion à cause de la similitude avec le passé récent.

En plus, en italien on utilise quatre types du subjonctif (congiuntivo) alors qu'en français on en utilise deux même s'il y en a quatre aussi. Leur emploi est différent car, en italien, on utilise congiuntivo avec les verbes d'opinion comme pensare ou credere pendant qu'en français le subjonctif est utilisé avec les verbes penser et croire seulement dans les questions et les négations.

En outre, l'emploi du passé simple et du passato remoto se diffère parce que le passé simple est utilisé pour décrire des événements historiques et on l'emploie dans la langue écrite pendant que le passato remoto fonctionne aussi dans la langue parlée et peut être utilisé pour raconter des faits récents si le locuteur ne ressent pas de liens émotifs avec ces faits.

Une autre différence concerne les phrases hypothétiques où, en français, après $s i$, il est interdit d'utiliser le futur pendant qu'en italien, c'est possible :

\begin{tabular}{|c|c|}
\hline \multicolumn{1}{|c|}{ Phrase française } & Phrase italienne \\
\hline S'il fait beau demain, on ira à la campagne. & Sefarà bel tempo domani, andremo in campagna. \\
\hline
\end{tabular}

La même conjonction si provoque des problèmes mais cette question sera analysée plus tard, dans le paragraphe sur les faux amis.

Pour conclure ce paragraphe, il faut ajouter qu'en français le verbe être crée les formes composées avec l'auxiliaire avoir alors qu'en italien essere crée ces formes avec l'auxiliaire essere :

\begin{tabular}{|l|l|}
\hline \multicolumn{1}{|c|}{ Phrase française } & Phrase italienne \\
\hline J'ai été en France l'année dernière. & Sono stata in Francia l'anno scorso. \\
\hline
\end{tabular}

Puisque les formes composées sont nombreuses et fréquentes, on peut facilement imaginer le nombre d'erreurs de ce type.

e. La formation du superlatif dans lequel en français on répète l'article alors qu'en italien on ne le répète pas : 


\begin{tabular}{|l|l|}
\hline \multicolumn{1}{|c|}{ Superlatif français } & \multicolumn{1}{c|}{ Superlatif italien } \\
\hline La fête la plus importante & La festa più importante \\
\hline
\end{tabular}

Les erreurs qui concernent la formation du superlatif, soit en italien, soit en français, sont très fréquentes chez les étudiants qui calquent la structure de l'autre langue sans tenir compte des différences.

f. L'emploi des pronoms personnels : il s'agit de la nécessité d'introduire le pronom personnel qui indique le sujet de la phrase dans la langue française pendant que dans la langue italienne, grâce à la conjugaison développée, il n'est pas obligatoire d'introduire le pronom dans chaque phrase. En conséquence, les étudiants ont tendance à répéter le pronom en italien, ce qui rend le style lourd (dans le cas de la langue française, on observe l'omission du pronom, aussi dans les expressions comme *est nécessaire de le faire à l'exemple de è necessario farlo).

Les erreurs mentionnées ci-dessus ont un caractère grammatical dans le sens que l'on applique les règles grammaticales d'une langue dans l'autre.

2.2.3. L'attribution au nom italien du genre de son équivalent français est un autre type d'erreurs. Évidemment, très souvent, le genre des deux noms est le même mais ce fait incite à élargir cette règle à tous les noms, ce qui provoque des erreurs. Parmi les cas les plus fréquents, on peut évoquer les paires de noms suivantes :

\begin{tabular}{|l|l|}
\hline \multicolumn{1}{|c|}{ Nom italien } & \multicolumn{1}{c|}{ Nom français } \\
\hline Un errore $(m)$. & Une erreur $(f)$. \\
\hline Un mare $(m)$. & Une mer $(f)$. \\
\hline Un periodo $(m)$. & Une période $(f)$. \\
\hline Un colore $(m)$. & Une couleur $(f)$. \\
\hline Una mancanza $(f)$. & Un manque $(m)$. \\
\hline
\end{tabular}

Il est inutile d'expliquer comment la différence de genre entre les mots très semblable en forme et identique en sens s'avère dangereuse et pourquoi elle cause tant de problèmes si les deux noms ont des genres différents.

2.2.4. Les fautes d'orthographe sont un autre problème fréquent, même si elles ne gênent pas dans la communication orale. Toutefois, dans le cas des étudiants en philologie aucune erreur ne peut être tolérée. Les exemples de l'influence de la langue française sur l'orthographe italienne sont: *lui a (au lieu de lui ha), *est (è), *et (e), *à (a), *corriggere (correggere), *confirmare (confermare), *produire (produrre), *fromaggio (formaggio), *quatro (quattro) et beaucoup d'autres. 
2.2.5. La création de mots inexistants en italien d'après le modèle français est un phénomène très caractéristique pour les étudiants en philologie qui sont en mesure de créer des mots tous seuls ou d'ajuster les mots d'une langue aux règles de l'autre langue. Tout cet effort ne sert à rien car ces structures n'appartiennent pas au lexique de la langue d'arrivée. Parmi les exemples rencontrés on peut citer les créations suivantes :

- Mangiavo ecutando la musica où le verbe français écouter est employé en italien au lieu du verbe ascoltare (le sens de la phrase est suivant : Je mangeais en écoutant la musique)

- terminazione inspiré par le mot français terminaison pendant qu'en italien fonctionne le terme desinenza

- Allemania créé d'après Allemagne au lieu de Germania

- concombre inspiré par concombre au lieu de cetriolo

- rincontro au lieu de incontro (rencontre), c'est une erreur très fréquente

- il giorno delle famme au lieu de il giorno delle donne inspiré par la journée de la femme

- di tempo in tempo (source : de temps en temps) au lieu de di tanto in tanto

- esplicazione (explication) au lieu de spiegazione et beaucoup d'autres.

Leur existence s'explique par deux facteurs : par le fait de ne pas connaître le terme et la nécessité de le créer (le processus plus ou moins conscient) et par la conviction qu'un tel mot existe vraiment, ce qui conduit à son emploi sans réflexion.

2.2.6. La dernière catégorie d'erreurs mentionnées, ce sont les erreurs liées à l'existence des faux amis. Évidemment, dans la langue italienne et dans la langue française, il y a un grand nombre de mots semblables du point de vue de la forme et du sens mais il y a aussi des mots qui, malgré la similitude de la forme, sont différents en ce qui concerne leurs sens. Nous avons observé, entre autres, les exemples suivants de faux amis.

\begin{tabular}{|l|l|l|}
\hline \multicolumn{1}{|c|}{$\begin{array}{c}\text { Phrases avec mots italiens aux } \\
\text { sens erronés }\end{array}$} & \multicolumn{1}{|c|}{$\begin{array}{c}\text { Phrases avec mots italiens } \\
\text { corrects }\end{array}$} & $\begin{array}{c}\text { Source française de } \\
\text { l'erreur }\end{array}$ \\
\hline Ho fermato la porta. & Ho chiuso la porta. & fermer \\
\hline $\begin{array}{l}\text { Vado alla festa dell'anniversario } \\
\text { di Marco. }\end{array}$ & $\begin{array}{l}\text { Vado alla festa del compleanno } \\
\text { di Marco. }\end{array}$ & anniversaire \\
\hline $\begin{array}{l}\text { I miei parenti sono ancora } \\
\text { giovani. }\end{array}$ & $\begin{array}{l}\text { I miei genitori sono ancora } \\
\text { giovani. }\end{array}$ & parents \\
\hline Sono assai bella. & Sono abbastanza bella. & assez \\
\hline Non si deve fare anzi. & Non si deve fare cosi. & ainsi \\
\hline Mio padre ha avuto un accidente. & Mio padre ha avuto un incidente. & accident \\
\hline
\end{tabular}

Une autre confusion, très fréquente, de ce type est celle liée à la fausse équivalence entre l'expression française c'est et l'expression italienne c'è. En réalité, la correspondance est différente : 


\begin{tabular}{|c|c|}
\hline Expression française & Équivalent italien \\
\hline$c^{\prime}$ 'est & $\grave{e}$ \\
\hline il $y a$ & $c^{\prime} \grave{e}$ \\
\hline
\end{tabular}

L'inclination à l'emploi de la forme italienne c'è au lieu de è peut avoir, au-delà de la valeur interférentielle, aussi un caractère psychologique, car il peut sembler impossible de présenter quelqu'un ou quelque chose avec une seule lettre.

Un autre exemple de ce type, c'est la confusion avec le pronom personnel se et la conjonction si qui ont leurs équivalents en croix :

\begin{tabular}{|c|c|}
\hline Élément français & Équivalent italien \\
\hline se (Elle se lève tard.) & si (Lei si alza tardi.) \\
\hline si (Si tu veux...) & se (Se vuoi...) \\
\hline
\end{tabular}

La confusion suivante de ce type est liée aux articles et aux pronoms qui sont confondus dans les deux langues :

\begin{tabular}{|c|c|}
\hline Élément français & Équivalent italien \\
\hline$l e$ & $i l$ \\
\hline$l e s(f)$. & $l e$ \\
\hline$i l$ & lui \\
\hline
\end{tabular}

Une autre difficulté réside dans l'emploi du pronom italien ne qui est souvent traité comme la négation pendant qu'il est l'équivalent du pronom français en :

\begin{tabular}{|c|c|}
\hline Élément français & Équivalent italien \\
\hline$e n$ & $n e$ \\
\hline$n e$ & non \\
\hline
\end{tabular}

Il est probable que l'identification du pronom ne avec la négation est liée non seulement à la forme française, mais aussi à la forme du pronom italien qui est tellement persuasive, car ce pronom est traité comme la négation aussi par les personnes qui ne connaissent pas la langue française.

\section{SOLUTIONS ET CONCLUSIONS POUR L'ENSEIGNANT}

Dans la partie précédente de cette analyse, nous avons énuméré et illustré quelques types d'erreurs commises par les étudiants. À ce moment-là, il faudrait trouver les solutions pour les éliminer ou, au moins, pour réduire leur nombre. Les linguistes proposent beaucoup de solutions à introduire par l'enseignant. Nous pouvons évo- 
quer les propositions de bases psychologiques de Komorowska (1980 : 120-128) qui conseille la répétition continue des structures à apprendre pour faciliter leur mémorisation (dans cette méthode, l'effort de l'apprenant est indispensable). Dans le cas des problèmes de l'interférence entre deux formes, on suggère la comparaison de l'une avec l'autre pour souligner les différences de leur sens ou leur emploi (c'est le rôle de l'enseignant). Les formes moins compliquées devraient être comparées ensemble alors que celles plus difficiles devraient apparaître séparément. Il est encore plus avantageux d'associer les structures au contexte situationnel dans lequel elles sont employées (de nouveau, c'est le devoir de l'enseignant). Selon Rivers (1972 : 39), l'enseignant devrait avertir ses étudiants sur le fait que la structure donnée peut causer des erreurs. Odlin (1989 : 161-163) souligne de sa part l'utilité du contraste qui aide à noter et à comprendre les différences entre les structures dans deux langues.

Un autre élément très important est le contact avec la culture du pays dont la langue que l'on apprend est parlée. Aujourd'hui, lorsque l'on apprend une langue étrangère pour communiquer et pas pour lire la littérature dans la version originale, il faut se concentrer surtout sur les éléments de la culture de la vie quotidienne (Grucza, 1989 : 42-44). La connaissance de la culture étrangère est d'autant plus importante que, souvent, c'est l'erreur culturelle et non grammaticale ou lexicale qui crée l'impression de manque de politesse chez l'interlocuteur. Évidemment, le séjour dans le pays où on parle la langue étudiée améliore sa maîtrise à tous les niveaux, de la prononciation, en passant par le lexique pour finir à la connaissance des coutumes.

Dans le cas des enfants provenant de familles bilingues, il est nécessaire de séparer les rôles linguistiques des parents, c'est-à-dire la mère doit communiquer avec les enfants seulement dans sa langue natale et le père doit communiquer uniquement dans la sienne pour que les enfants associent chaque langue en isolation, avec un des parents et ainsi ils ne confondent pas les systèmes (Grucza, 1981 : 34). D'une manière analogue, l'enseignant ne devrait pas mélanger les langues non plus, surtout pas dans la même phrase, les diverses parties de la leçon devraient être nettement séparées linguistiquement.

En revenant aux stratégies pour l'enseignant, il est important que celui-ci incite ses étudiants à reconnaître les structures «dangereuses » et à s'autocorriger. Il est nécessaire que l'enseignant contraste la forme correcte avec celle erronée. En plus, il doit expliquer comment une telle erreur provoque la confusion dans la communication. Quand même, il faut se rendre compte que les erreurs sont une partie inséparable de l'apprentissage et il faut les traiter comme telles (Wilczyńska, $1980: 143$ ).

Nous tirons certaines conclusions de l'expérience du travail comme enseignant de l'italien aux personnes qui connaissent déjà la langue française. Lorsque l'on introduit une règle de grammaire en italien, il est conseillable de la contraster avec la règle française pour souligner les points communs et les différences de l'emploi. L'introduction du lexique exige la même procédure : il faut expliquer p.ex. que, dans la 
prononciation du mot français septembre, il y a le lien de consonnes -pt- pendant que dans le mot italien settembre il y a -tt-. Même s'il semble exagéré (l'étudiant sait lire, n'est ce pas ?!), il faut attirer l'attention de l'apprenant sur les détails car beaucoup de personnes ne les remarquent pas.

Plus tard, quand une structure est employée, l'enseignant doit faire attention et si l'étudiant hésite ou commet une erreur, il faut commenter la situation avec des mots de type : «Ce n'est pas le français, mais l'italien » quand les règles sont différentes, ou avec les mots « C'est comme en français » si les règles sont analogues. Une des solutions évoquées ci-dessus prétend qu'il ne faut pas mélanger les langues mais nous sommes d'avis qu'un étudiant avancé en français peut apprendre sans problèmes une règle italienne en la contrastant avec celle française. Ce procédé facilite l'apprentissage car il est plus facile de mémoriser une règle par analogie avec une autre langue romane que sans aucune aide (la connaissance du polonais n'aide en rien vue la différence des systèmes grammaticaux de deux langues). En même temps, on répète la structure française, ce qui peut prévenir l'interférence pas seulement en italien, mais plus tard en français, aussi.

Dans le cas de l'emploi des mots et des structures français dans la langue italienne (il s'agit surtout de mais au lieu de ma et de formation des structures comme *è difficile di fare), la solution est la répétition des structures correctes : l'enseignant doit simplement corriger ses étudiants quand ils commettent des erreurs. Avec un peu de pratique, quand ces structures deviennent fortes, l'interférence sera moins probable (dans cette situation, ce qui vaut c'est la règle de l'influence de la structure forte sur la structure faible).

Nous sommes convaincue qu'au niveau avancé des deux langues romanes en question le plus difficile est de connaître d'une manière exacte l'orthographe des mots et que les règles de grammaire ne causent pas autant de problèmes. Surtout le français, avec ses accents et la prononciation qui peut correspondre à plus d'une graphie, est un défi. Pour cette raison, il faut transmettre aux étudiants la conviction qu'ils sont capables de parler les langues, leur donner de la confiance en eux-mêmes, mais, en même temps, il faut enseigner aux étudiants de consulter le dictionnaire en cas de doutes et de ne pas trop croire aux fausses similitudes. En bref, il est avantageux de leur montrer les similitudes qui facilitent l'apprentissage mais il est nécessaire de souligner le fait que l'italien et le français restent toujours des langues distinctes.

En outre, nous observons chez les étudiants le recours constant à l'autre langue, c'est-à-dire ils pensent en français en parlant l'italien ce qui cause un grand nombre d'erreurs. Pour le réduire, les étudiants doivent savoir dans quelle langue ils pensent et ils doivent se rendre compte que l'aide de l'autre langue est favorable seulement dans la situation où elle est consciente. L'interférence inconsciente provoque d'habitude des erreurs.

Toutes les considérations mentionnées ci-dessus peuvent être résumées en une phrase : la parenté entre le français et l'italien facilite sûrement le processus de l'ap- 
prentissage mais il est nécessaire que l'on maintienne la vigilance et que l'on vérifie ce que l'on dit ou écrit. C'est le rôle de l'enseignant d'implanter cette attitude chez les étudiants.

\section{BIBLIOGRAPHIE}

Grucza F. (1981). Glottodydaktyczne implikacje bilingwizmu, in : Bilingwizm a Glottodydaktyka. Materiały z V Sympozjum zorganizowanego przez Instytut Lingwistyki Stosowanej UW, Biatowieża 26-28 maja 1977 (pp. 9-35), Warszawa : Wydawnictwa UW.

Grucza F. (1989). Język a kultura, bilingwizm a bikulturyzm : lingwistyczne i glottodydaktyczne aspekty interlingwalnych i interkulturowych różnic oraz zbieżności, in : Bilingwizm, bikulturyzm, implikacje glottodydaktyczne. Materiaty z XII Sympozjum zorganizowanego przez Instytut Lingwistyki Stosowanej UW, Zaborów, 18-20 września 1986, (pp. 9-51), Warszawa : Wydawnictwo UW.

Komorowska H. (1980). Nauczanie gramatyki języka obcego a interferencja, Warszawa : WOSP.

Odlin T. (1989), Language Transfer. Cross-linguistic influence in language learning, Cambridge University Press.

Rivers W.M. (1972). Speaking in Many Tongues : Essays in Foreign-Language Teaching, Rowley : Newbury House Publishers.

Szulc A. (1994). Słownik dydaktyki języków obcych, Warszawa : Wydawnictwo Naukowe PWN.

Weinreich U. (1953). Languages in contact. Findings and problems, the Hague : Mouton Publishers.

Wilczyńska W. (1980), Pour une nouvelle conception de l'erreur dans la pédagogie d'une langue étrangère, Studia Romanica Posnaniensia, VII, 137-144. 